\section{P2-41 PREVALENCE OF SMOKING AND ITS ASSOCIATION WITH MORTALITY IN CHINA: FINDINGS OF THE KADOORIE BIOBANK STUDY OF 0.5 MILLION PEOPLE}

doi:10.1136/jech.2011.142976h.77

${ }^{1} Z$ Chen, ${ }^{*}{ }^{2} Y$ Guo, ${ }^{1} M$ Smith, ${ }^{3} J$ Chen, ${ }^{1} R$ Collins, ${ }^{1} R$ Peto, ${ }^{2} \mathrm{~L}$ Li. ${ }^{1}$ Clinical Trial Service Unit \& Epidemiological Studies Unit, University of Oxford, Oxford, UK; ${ }^{2}$ Chinese Academy of Medical Sciences, Beijing, China; ${ }^{3}$ China Centre for Disease Control and Prevention, Beijing, China

Background Over the past few decades, there has been a rapid increase in cigarette consumption in China. Large prospective studies are needed to monitor the evolution over the next few decades of the resultant epidemic of tobacco-related deaths in China. Methods During 2004-2008 the Kadoorie Biobank Study recruited 510000 adults aged 30-79 from 10 geographically defined regions across China. By Oct 2010, 10000 deaths had been recorded. Cox regression was used to relate smoking to cause-specific mortality.

Results At baseline, the prevalence of ever smoking regularly was much higher in men (74\%) than in women (3\%). Among men, the smoking prevalence varied little by area and age but among women, it was associated inversely with year of birth, reflecting mainly the progressive decline in the uptake rate of smoking among younger women over the past few decades. Among men, the overall mortality of ever regular smokers was significantly higher than that of never smokers (adjusted RR 1.32, 95\% CI 1.21 to 1.43). There were significant excess mortalities from total cancer, CVD and respiratory disease in male smokers. In urban men, the RR of ever regular smoking was the highest (RR 2.2, 95\% CI 2.01 to 2.44) for those who started smoking before age 20 years, suggesting that over $50 \%$ of such deaths were caused by smoking.

Conclusion Smoking is already a major cause of premature death in China. The findings among urban men indicate the proportion of male deaths that could eventually be caused by smoking in China as whole.

\section{P2-42 RISK AND SOCIAL DETERMINANTS OF ANGINA IN OLDER PEOPLE IN CHINA: ROSE ANGINA QUESTIONNAIRE AND DOCTOR-DIAGNOSIS APPROACHES}

doi:10.1136/jech.2011.142976h.78

\author{
${ }^{1,2}$ R Chen. ${ }^{*}$ Anhui Medical University, Hefei, China; ${ }^{2}$ Unviersity of Wolverhampton, \\ Wolverhampton, UK
}

Background The risk of angina and its social determinants in the elderly in China are not well documented. We determined the prevalence and socio-economic predictors of angina in Chinese elderly using the Rose Angina questionnaire and doctor-diagnosed of angina. Methods Using a standard interview method, we examined random samples of 4314 participants aged $\geq 60$ years in Guangdong, Heilongjiang, Shanghai and Shanxi provinces during 2008-2009. An angina diagnosis was based on the Rose Angina questionnaire and a doctor-diagnosis. An age-sex adjusted logistic regression model was employed to determine the association between angina and sociodemographic variables.

Results The world age-standardised prevalence of angina using the Rose Angina questionnaire $(n=246)$ was $5.74 \%(95 \%$ CI $5.00 \%$ to $6.47 \%$ ); in men $3.69 \%$ (2.75\% to $4.63 \%$ ), in women $7.22 \%$ (6.15\% to $8.29 \%)$. Corresponding values for doctor-diagnosed angina $(\mathrm{n}=282)$ were $6.54 \%(5.76 \%$ to $7.32 \%)$; in men $5.08 \%$ (4.00\% to $6.17 \%$ ), in women $7.67 \%(6.56 \%$ to $8.77 \%)$. There was a fair agreement between two diagnoses (overall $\kappa=0.38$, in men 0.35 , in women 0.40 ; all $\mathrm{p}<0.001$ ). For both measures the likelihood of a diagnosis of angina was greater in women, heavier body weight, higher BMI, cigarette smoking and hypertension. Doctor diagnosed angina was associated with higher levels of educational attainment and higher occupation class, living in an urban area, taller height, larger waist circumference and diabetes. A diagnosis of angina based on the Rose Angina questionnaire was associated with low income only.

Conclusions Older people in China may have a low prevalence of angina. The association between an angina diagnosis based on the Rose Angina questionnaire and low income, and between a doctor diagnosis of angina and high socioeconomic status and urban living suggests that significant health and care inequalities in cardiovascular medicine exist in China.

\section{P2-43 WITHDRAWN}

\section{P2-44 BURDEN OF STROKE AND ITS RELATED DISABILITIES IN A RURAL COMMUNITY OF BANGLADESH}

doi:10.1136/jech.2011.142976h.79

${ }^{1} \mathrm{~S}$ Choudhury, ${ }^{2} \mathrm{~J}$ Ahmed, ${ }^{2} \mathrm{M}$ Zaman, ${ }^{1} \mathrm{M}$ Sobhan, ${ }^{3} \mathrm{~A}$ Hussain. ${ }^{1}$ National Heart Foundation Hospital \& Research Institute, Dhaka, Bangladesh; ${ }^{2}$ Ekhlaspur Center of Health, Motlob North, Bangladesh; ${ }^{3}$ Bangabandhu Sheikh Mujib Medical University, Dhaka, Bangladesh

Introduction Demographic changes and increased exposure to stroke risk factors are expected to give rise to high stroke burden in countries like Bangladesh. However data on stroke prevalence and its related disabilities in rural community of Bangladesh are scarce. Methods A survey was done in 2006 in a rural population to identify stroke cases living in the community. A validated questionnaire for detection of stroke was used to identify probable cases by visiting households by trained interviewers. Identified probable cases were seen by physicians for confirmation. Physicians also determined the levels of disability according to Modified Rankin Scale.

Results Interviewers identified 61 people as probable stroke cases among the 4080 people aged 30 years or above. Physicians confirmed 26 cases of stroke (men: 21, women: 5) giving a stroke prevalence 64 per 10000 (95\% CI 42 to 93). Mean age of onset of stroke was 57 years (95\% CI 50 to 64). Various types of residual disabilities such as hemiplegia, paraplegia, facial weakness were noted among the $85 \%$ of the patients. The median modified Rankin Scale score was five, which suggests that $50 \%$ of the cases had moderate disability such as unable to walk without assistance and unable to attend to own bodily needs without assistance.

Conclusion Number of stroke cases living in the community with disability was high. Appropriate care and rehabilitation programme along with measures for reduction of population risk factor levels are needed.

\section{P2-45 PERIODONTAL DISEASE CONTRIBUTES TO THE OCCURRENCE OF CARDIOVASCULAR EVENTS? A META- ANALYSIS OF CASE CONTROL STUDIES}

doi:10.1136/jech.2011.142976h.80

${ }^{1} \mathrm{~J}$ M F Coelho, ${ }^{* 1}$ I S Gomes-Filho, ${ }^{3} \mathrm{M}$ L Barreto, ${ }^{3} \mathrm{~A}$ Guimarães, ${ }^{5} \mathrm{P}$ Lotufo, ${ }^{3} \mathrm{~A}$ F Coelho, ${ }^{1} \mathrm{~J}$ S Passos, ${ }^{4} \mathrm{P}$ B Barbosa, ${ }^{2} \mathrm{~S}$ S Cruz, ${ }^{1}$ S C Trindade. ${ }^{1}$ Universidae Estadual de Feira de Santana, Feira de Santana, Bahia, Brazil, ${ }^{2}$ Universidade Federl do Vale do São Francisco, Petrolina, Pernambuco, Brazil, ${ }^{3}$ Universidae Federal da Bahia, Salvdor, Bahia, Brazil; "Unação Baiana para Desenvolvimento da Ciência, Salvador, Bahia, Brazil; ${ }^{5}$ Universidade de São Paulo, São Paulo, São Paulo, Brazil

Introduction We carried out a meta-analysis to determine the association between exposure to $\mathrm{PD}$ and ischaemic $\mathrm{CDH}$.

Material and methods Studies published case-control between 1999 and 2009 were obtained from the search site PUBMED/MEDLINE, Lilacs e Google. The articles included report results of observational studies, case-control on the association between PD and DCVI, where the DP should have been diagnosed by clinical parameters 